\title{
Presence of autoantibodies against HeLa small nuclear ribonucleoproteins in chagasic and non-chagasic cardiac patients
}

M.C. Bosetto, M.S. Peixoto,

L.I.R. de Castro and R.M.B. Cicarelli
Departamento de Ciências Biológicas, Faculdade de Ciências Farmacêuticas, Universidade Estadual Paulista, Araraquara, SP, Brasil

\section{Correspondence \\ R.M.B. Cicarelli \\ Departamento de Ciências Biológicas \\ FCF, Universidade Estadual Paulista \\ Rod. Araraquara-Jaú, km 01 \\ 14801-902 Araraquara, SP \\ Brasil \\ Fax: +55-16-3301-6840 \\ E-mail: cicarell@fcfar.unesp.br}

\section{Research supported by FAPESP} (Nos. 97/01891-1 and 99/11393-4).

M.C. Bosetto was supported by CAPES and M.S. Peixoto was supported by FAPESP.

Received February 4, 2003 Accepted October 14, 2003

\begin{abstract}
We detected anti-human small nuclear ribonucleoprotein (snRNP) autoantibodies in chagasic patients by different immunological methods using HeLa snRNPs. ELISA with Trypanosoma cruzi total lysate antigen or HeLa human U small nuclear ribonucleoproteins (UsnRNPs) followed by incubation with sera from chronic chagasic and nonchagasic cardiac patients was used to screen and compare serum reactivity. Western blot analysis using a $T$. cruzi total cell extract was also performed in order to select some sera for Western blot and immunoprecipitation assays with HeLa nuclear extract. ELISA showed that 73 and $95 \%$ of chronic chagasic sera reacted with HeLa UsnRNPs and $T$. cruzi antigens, respectively. The Western blot assay demonstrated that non-chagasic cardiac sera reacted with high molecular weight proteins present in $T$. cruzi total extract, probably explaining the $31 \%$ reactivity found by ELISA. However, these sera reacted weakly with HeLa UsnRNPs, in contrast to the chagasic sera, which showed autoantibodies with human Sm (from Stefanie Smith, the first patient in whom this activity was identified) proteins (B/B', D1, D2, D3, E, F, and G UsnRNP). Immunoprecipitation reactions using HeLa nuclear extracts confirmed the reactivity of chagasic sera and human UsnRNA/RNPs, while the other sera reacted weakly only with U1snRNP. These findings agree with previously reported data, thus supporting the idea of the presence of autoimmune antibodies in chagasic patients. Interestingly, non-chagasic cardiac sera also showed reactivity with $T$. cruzi antigen and HeLa UsnRNPs, which suggests that individuals with heart disease of unknown etiology may develop autoimmune antibodies at any time. The detection of UsnRNP autoantibodies in chagasic patients might contribute to our understanding of how they develop upon initial T. cruzi infection.
\end{abstract}

Key words

- Chagas' disease

- Autoantibodies

- Small nuclear

ribonucleoprotein

- Trypanosoma cruzi

- HeLa cells

- Cross-reactivity 


\section{Introduction}

In Brazil and Latin America, Chagas' disease still represents a serious social and medical problem since this endemic disease affects about 8 millions of mostly poor inhabitants living under precarious housing conditions. The disease has three phases: acute, latent and chronic. Myocarditis is a visceral involvement present in all phases. In the acute phase, characterized by high levels of parasitemia, cases may range from asymptomatic to oligosymptomatic and to serious and even fatal, although death occurs in less than $3.5 \%$ of chagasic individuals. This phase is characterized by exponential parasite growth, triggering an intense immunological response. The latent phase follows the acute one and precedes the chronic one for about 10 to 20 years. At the end of the chronic phase, the worst clinical manifestations appear, with the occurrence of cutaneous eruptions, megaesophagus and megacolon, cardiomegaly, and occasionally hepatosplenomegaly.

Sera from chagasic individuals are characterized by the presence of anti-Trypanosoma cruzi antibodies accompanied in some cases by detectable autoantibodies (1-3). These sera present antibodies that react with $\mathrm{P}$ ribosomal proteins $(4,5)$ and are considered to be specific serological markers for systemic lupus erythematosus (SLE) (6), but without any clear correlation between individual clinical situation and the presence of circulating autoantibodies. Nonetheless, the nature of antigen targeted was never established. Many autoimmune reactions detected in chagasic individuals can be explained by the similarity between the proteins of host and parasite, which induce autoantibodies by molecular cross-reactions with cardiac and nervous tissue structures (7-13). Such anti-heart immune response might arise by molecular mimicry among some $T$. cruzi antigens homologous to cardiac proteins or by secondary cardiac proteins from the myo- carditis caused by the parasite during the acute phases (14). A human cardiac myosin heavy chain heart-specific epitope (14421447 residues, AAALDK) has also been detected, showing molecular mimicry with secondary epitopes (AAAGDK hexapeptide) of the B13 immunodominant recombinant protein of $T$. cruzi (15).

A theory proposed to explain autoimmunity suggests that the phenomenon may be initiated by a foreign protein, which might share epitopes with some human proteins. This may cause a primary autoimmune reaction with the appearance of initial autoantibodies. These antibodies may react with human factors such as ribonucleoproteins (RNPs), thus causing a secondary reaction that leads to the appearance of anti-RNP autoantibodies. Bach-Elias et al. (16) suggested that the proteins of the T. cruzi parasite, destroyed by the host immune system, might be presented again, thus triggering autoantibody production. The presence of common epitopes in T. cruzi and human $\mathrm{U}$ small nuclear RNPs (UsnRNP) has been detected. Other experimental data involving molecular mimicry support the hypothesis of chronic cardiac autoimmunity pathogenesis as well as irreversible digestive lesions. There are at least two non-mutually exclusive explanations for the generation of autoimmunity: 1) the parasite infection disturbs immunoregulation, leading to the loss of self antigen tolerance, and 2) immune recognition of $T$. cruzi antigens, which crossreact with some types of mammalian antigens, causes autoreactivity of B or T lymphocyte clones, which proliferate and lead to autoimmune lesions in chagasic patients (16). Since the etiology of Chagas' disease is well known, this disease has become an important model for autoimmunity studies and may also permit the study of the onset of autoantibody production.

In the present study, the detection of human anti-UsnRNP autoantibodies in chagasic patient sera was confirmed by different 
immunological methods. The detection of these antibodies should facilitate future understanding about how these antibodies developed upon initial T. cruzi infection.

\section{Material and Methods}

\section{Chagasic and non-chagasic sera}

Serum samples from chronic chagasic individuals with cardiac involvement, from non-chagasic cardiac patients and from normal individuals, and SLE sera were kindly provided by the Departamento de Cardiologia, Hospital das Clínicas, Faculdade de Medicina de Ribeirão Preto, Universidade de São Paulo, Ribeirão Preto, SP, Brazil. The study was approved by the Research Ethics Committee of FCF, UNESP.

Trypanosoma cruzi cell culture and maintenance ( $Y$ strain)

Epimastigote forms of T. cruzi (Y strain) were established and cultured in liver infusion tryptose medium at $25^{\circ} \mathrm{C}$ for 15 days. Five hundred milliliters of culture $\left(2 \times 10^{9}\right.$ total cells) was used to prepare the total lysate antigen for enzyme-linked immunosorbent assay (ELISA) and Western blot analysis.

\section{HeLa nuclear extracts}

HeLa nuclear extracts were prepared by the method of Dignam et al. (17), aliquoted and stored at $-80^{\circ} \mathrm{C}$ until the time for use. The extracts were treated with phenol/chloroform/isoamylalcohol (PCA, 25:24:1, v/v; GibcoBRL, Grand Island, NY, USA), vortexed and centrifuged at 10,000 $\mathrm{g}$ for $5 \mathrm{~min}$. The aqueous phase (snRNAs) was transferred to another tube and the same volume of cold acetone was added to the phenol phase. The preparation was mixed gently, incubated overnight at $-20^{\circ} \mathrm{C}$, and centrifuged at $10,000 \mathrm{~g}$ for $20 \mathrm{~min}$ at $4^{\circ} \mathrm{C}$. The pellet was washed with $70 \%$ ethanol, al- lowed to dry for a short time and resuspended in electrophoresis sample buffer $(50 \%$ glycerol, 10\% SDS, 25\% B-mercaptoethanol, 100 mM Tris-HCl, pH 6.8, $0.025 \%$ bromophenol blue).

\section{ELISA}

Microplates were coated overnight at $4^{\circ} \mathrm{C}$ with $1.0 \mu \mathrm{g}$ of antigen per well in $0.1 \mathrm{M}$ carbonate/bicarbonate buffer, $\mathrm{pH}$ 9.6. UsnRNPs isolated from HeLa nuclear extracts were kindly provided by Dr. Montserrat Bach-Elias (Consejo Superior de Investigaciones Científicas, Barcelona, Spain) and also used as antigen to coat the plates. The plates were washed once with PBS, $\mathrm{pH}$ 7.2, containing $0.05 \%$ Tween 20 (PBS/Tw) and incubated with blocking buffer (PBS/Tw plus $0.5 \%$ low fat milk) for $1 \mathrm{~h}$ at $37^{\circ} \mathrm{C}$. The plates were washed again and the sera diluted 1:100 in blocking buffer were added to the wells and incubated for $1 \mathrm{~h}$ at room temperature. The plates were washed three times and 50 $\mu l$ peroxidase-labeled anti-human immunoglobulin $\mathrm{G}$ (IgG) antibodies (GibcoBRL) diluted 1:10,000 in blocking buffer was added to each well and incubated for $1 \mathrm{~h}$ at room temperature. The plates were washed again and the color was developed by adding $50 \mu \mathrm{l}$ OPD to each well (GibcoBRL) $(0.4 \mu \mathrm{g} / \mathrm{ml}$ in citrate buffer containing $\mathrm{H}_{2} \mathrm{O}_{2}$ ). After $10 \mathrm{~min}$ of incubation at $37^{\circ} \mathrm{C}$ in the dark, absorbance at $492 \mathrm{~nm}$ was measured with an automated plate reader (BioRad). The cut-off values used in these assays were: high (0.16 and 0.40), medium (0.10 and 0.20) and low (0.02 and 0.01), when using HeLa UsnRNPs and T. cruzi lysate antigen, respectively. Normal human serum absorbance was below 0.001 .

\section{Western blotting}

Samples of T. cruzi total cell lysates or HeLa nuclear extract RNPs were electrophoresed under denaturing conditions on sodium dodecyl sulfate-polyacrylamide gel 
(5\% stacking gel, $10 \%$ separating gel). The samples were transferred to nitrocellulose membranes $\left(1 \mathrm{~h}, 100 \mathrm{~V}, 4^{\circ} \mathrm{C}\right)$, wrapped in aluminum foil and stored at $-20^{\circ} \mathrm{C}$ until use (18).

The nitrocellulose sheets were blocked using $\mathrm{PBS} / \mathrm{Tw} / 0.5 \%$ low fat milk (blocking solution) for $1 \mathrm{~h}$ at room temperature under

Table 1. Reactivity with Trypanosoma cruzi total extract of sera from patients with non-chagasic and chagasic heart disease.

\begin{tabular}{|c|c|c|c|c|}
\hline & \multicolumn{2}{|c|}{$\begin{array}{c}\text { Non-chagasic } \\
\text { sera }\end{array}$} & \multicolumn{2}{|c|}{$\begin{array}{c}\text { Chagasic } \\
\text { sera }\end{array}$} \\
\hline & Abs & $\%$ & Abs & $\%$ \\
\hline Low & 0.005 & 69 & 0.01 & 5 \\
\hline Medium & 0.250 & 24 & 0.30 & 13 \\
\hline High & 0.500 & 7 & 0.70 & 82 \\
\hline
\end{tabular}

Eighty-five sera from non-chagasic patients and 133 sera from chagasic patients were tested by ELISA using T. cruzi antigen and their degree of reactivity (low, medium and high) was determined. A chagasic serum with strong reactivity and normal human sera were used as positive and negative controls, respectively (data not shown). Mean absorbances (Abs) are given in the Table.

Table 2. Reactivity with HeLa human $U$ small nuclear ribonucleoproteins (UsnRNPs) of sera from patients with non-chagasic and chagasic heart disease.

\begin{tabular}{lllllr}
\hline & \multicolumn{2}{c}{$\begin{array}{c}\text { Non-chagasic } \\
\text { sera }\end{array}$} & & \multicolumn{2}{c}{$\begin{array}{c}\text { Chagasic } \\
\text { sera }\end{array}$} \\
\cline { 2 - 3 } \cline { 5 - 6 } & Abs & $\%$ & & Abs & $\%$ \\
\hline Low & 0.04 & 32 & & 0.05 & 27 \\
Medium & 0.12 & 56 & & 0.15 & 68 \\
High & 0.18 & 12 & & 0.25 & 5 \\
\hline
\end{tabular}

Thirty-six sera from chagasic patients and 22 sera from non-chagasic patients were tested by ELISA using HeLa UsnRNPs as antigen and their degree of reactivity (low, medium and high) was determined. A chagasic serum with strong reactivity and normal human sera were used as positive and negative controls, respectively (data not shown). Mean absorbances (Abs) are given in the Table. shaking, followed by washes (three times, 5 min each) with PBS/Tw. Nitrocellulose strips $(1 \mathrm{~cm})$ were cut and incubated for $16 \mathrm{~h}$ at $4^{\circ} \mathrm{C}$ with shaking with serum diluted 1:100 in blocking solution. After incubation, the strips were washed as described above and incubated with peroxidase-labeled anti-human IgG (Sigma, St. Louis, MO, USA) diluted 1:2000 in PBS/Tw or peroxidase-labeled protein A (Sigma; diluted 1:2000) for $1 \mathrm{~h}$ at room temperature followed by washing with 0.05 M Tris-HCl, $\mathrm{pH}$ 7.6, three times for 5 $\mathrm{min}$ and developed for $10 \mathrm{~min}$ with diaminobenzidine (GibcoBRL) diluted as suggested by the manufacturer, and the reaction was blocked with distilled water.

\section{Immunoprecipitation assays using HeLa nuclear extracts (19)}

Human sera were precipitated with an equal volume of $100 \%$ saturated ammonium sulfate solution and the precipitate washed twice with a $50 \%$ saturated solution. The precipitated IgGs were bound to protein ASepharose (GibcoBRL) overnight at $4^{\circ} \mathrm{C}$ and incubated with HeLa nuclear extract for $18 \mathrm{~h}$ at $4^{\circ} \mathrm{C}$ under rotation. The pellet was washed and treated with proteinase $\mathrm{K}(20 \mathrm{mg} / \mathrm{ml})$ for $15 \mathrm{~min}$ at $37^{\circ} \mathrm{C}$, followed by PCA extraction (v/v). The small RNAs (UsnRNAs) were precipitated with ethanol and submitted to electrophoresis on $10 \%$ denaturing urea/polyacrylamide/TBE buffer gel followed by silver staining.

\section{Results}

ELISA

Eighty-five sera from non-chagasic cardiac individuals and 133 sera from chagasic cardiac patients were analyzed by ELISA using $T$. cruzi antigen and then assessed for reactivity using a chagasic serum sample with a high degree of reactivity (average absorbance $>0.7$ ) as positive control. These 
results are shown in Table 1. As expected, among the non-chagasic cardiac sera, 69\% showed low reactivity with $T$. cruzi antigen (average absorbance $=0.005$ ), and only $7 \%$ of them showed cross-reactivity with this antigen (average absorbance $=0.5$ ). The cutoff points for high, medium and low reactivity were given in Material and Methods.

A set of 36 chagasic sera and 22 nonchagasic cardiac sera (medium and high reactivity) were analyzed by ELISA using $\mathrm{HeLa}$ UsnRNPs as antigen and the results are shown in Table 2. The absorbance values of nonchagasic sera were lower (see the mean absorbance values annotated in the Table) than those obtained with chagasic sera, probably because infection with the parasite can raise autoantibody levels.

Similar results were previously obtained by Bach-Elias et al. (16), who reported 73\% cross-reactivity of chagasic sera with HeLa UsnRNP antigens, thus supporting the idea of the presence of autoimmune antibodies in these individuals. However, it is interesting to note that non-chagasic cardiac sera, which showed high and medium reactivity with $T$. cruzi antigen, also reacted with $\mathrm{HeLa}$ UsnRNPs in $68 \%$ of cases. This may suggest that individuals with cardiac involvement of unknown etiology may somehow develop autoimmune antibodies, which could recognize some epitopes in the T. cruzi antigen.

\section{Western blot analysis}

In an attempt to understand the ELISA results in Table 1 the same sera were also tested by Western blot containing T. cruzi total cell extract as antigen, which showed different patterns of reactivity. Two sera from each reactivity group were chosen to illustrate the results presented in Figure 1, panel I (chagasic sera) and panel II (non-chagasic cardiac sera). These results demonstrate that non-chagasic cardiac sera only reacted with high molecular weight proteins, which are present in $T$. cruzi total extract, helping to explain the lower absorbance values obtained by ELISA, as well as the cross-reactivity among these non-chagasic cardiac sera.

In order to determine the cross-reactivity between chagasic sera and human UsnRNPs, we analyzed all chagasic sera by Western blot using HeLa UsnRNPs as antigen and compared them with SLE serum as positive

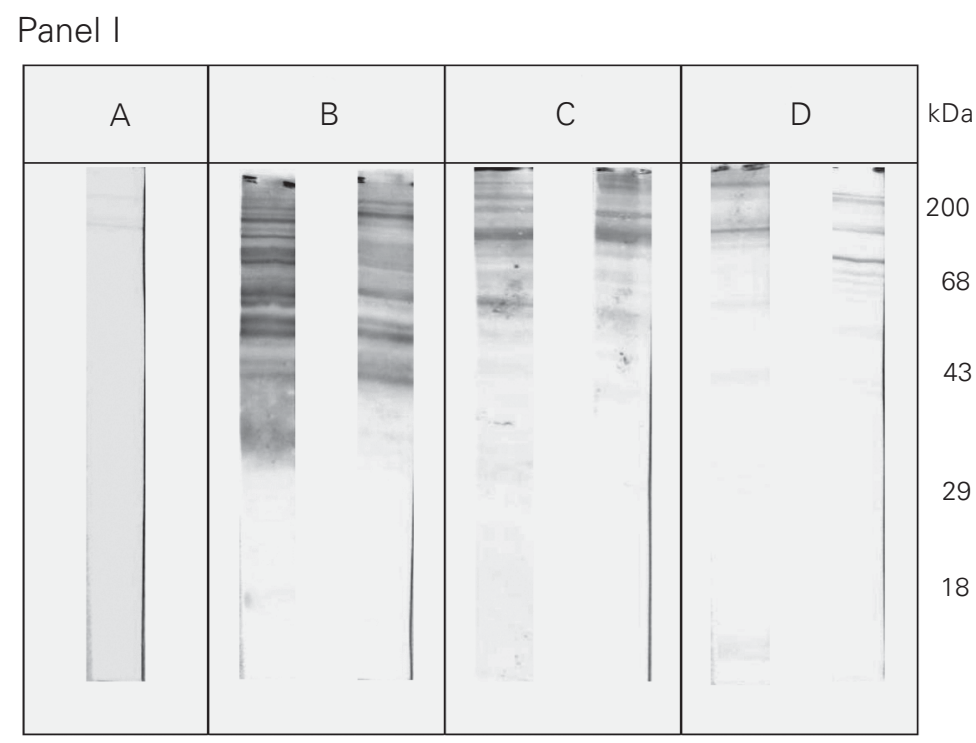

Panel II

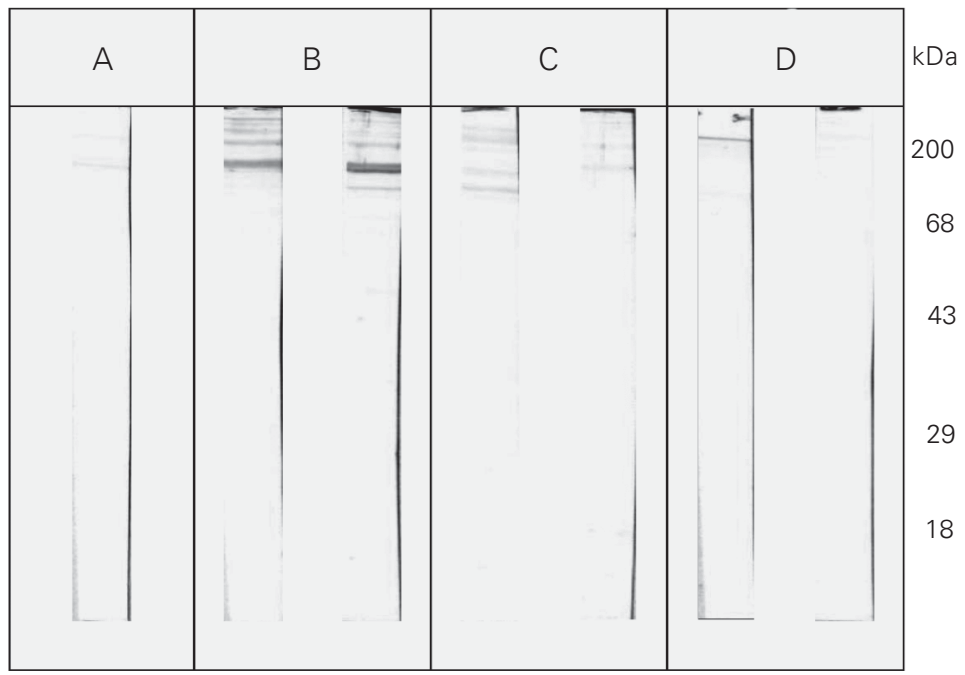

Figure 1. Western blot of sera from patients with chagasic (Panel I) and non-chagasic (Panel II) heart disease using T. cruzi total cell extract. Two sera from each group were chosen to illustrate the results. A, Normal human serum. B, Sera with high reactivity to ELISA. $C$, Sera with medium reactivity to ELISA. D, Sera with low reactivity to ELISA. The molecular weights are indicated in $\mathrm{kDa}$. 
control. Figure 2 shows one reaction for each serum to illustrate the results. Thus, these data confirm that chronic chagasic individuals develop autoantibodies against human Sm proteins (B/B', D1, D2, D3, E, F, and $\mathrm{G}$ UsnRNP), as mentioned elsewhere

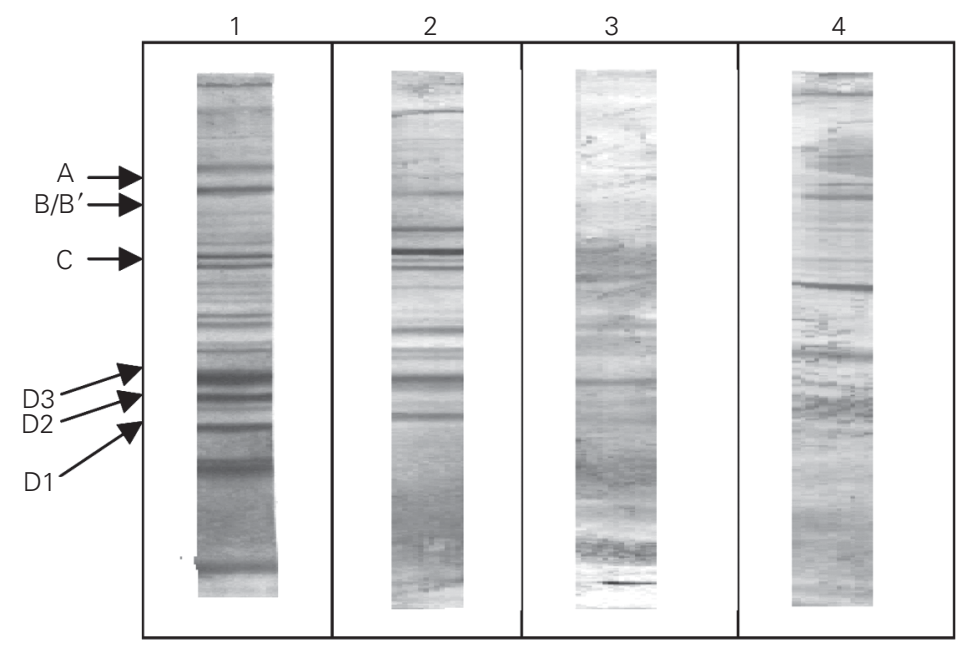

Figure 2. Western blot of serum from a patient with chagasic heart disease (lane 2 - high degree of reactivity in Figure 1, Panel I) and from a patient with non-chagasic heart disease (lane 3 - high degree of reactivity in Figure 1, Panel II) using HeLa UsnRNPs. Lane 1, Serum from a patient with systemic lupus erythematosus as positive control; lane 4, normal human serum. The typical human Sm proteins (A, B/B', C, D1, D2, D3) are indicated.

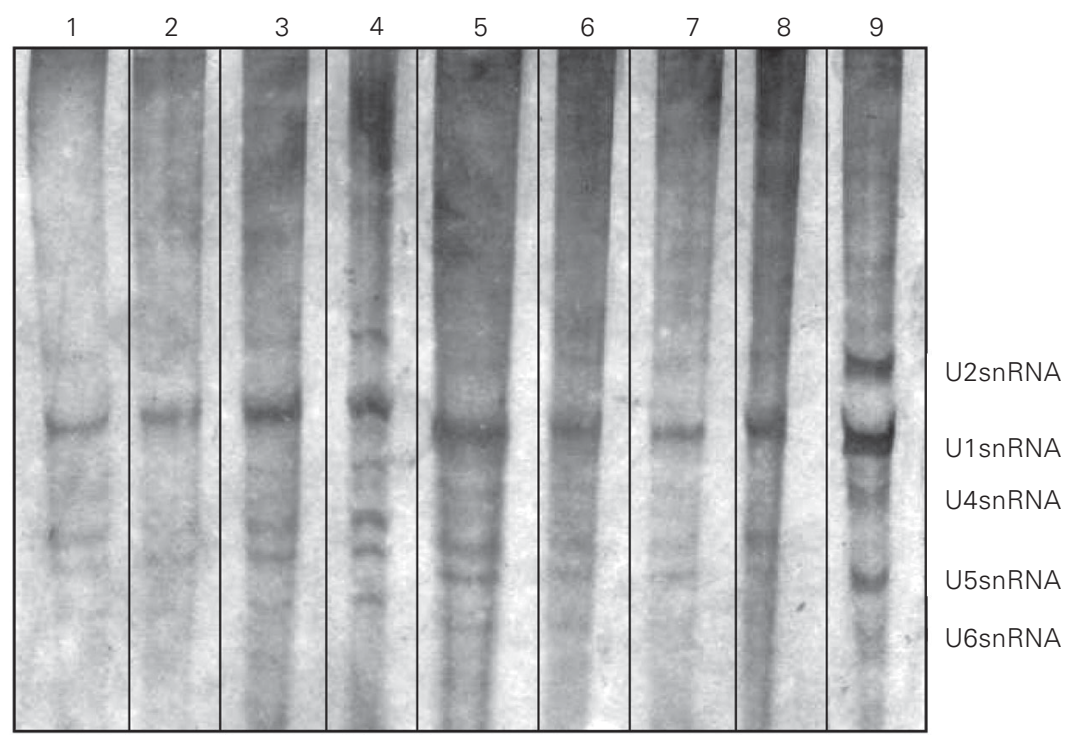

Figure 3. Immunoprecipitation of HeLa nuclear extract by human sera. Human small nuclear RNAs (UsnRNAs) were analyzed in 10\% urea/polyacrylamide gel/TBE [1X]. Lane 1, Normal human serum; lane 2, cardiac non-chagasic serum; lanes 3 through 8 , chagasic sera; lane 9, anti-m3G antibody used as positive control to characterize the HeLa UsnRNAs.
$(16,20)$. However, in the present experiments, the non-chagasic cardiac sera showed very faint bands similar to those for normal serum.

\section{Immunoprecipitation assays}

In an attempt to determine whether the previously tested antibodies were able to recognize human UsnRNA/RNPs, some sera were used in immunoprecipitation reactions with HeLa nuclear extracts, as described in Material and Methods. The results are shown in Figure 3 and demonstrate that chagasic sera, in fact, presented antibodies against different human UsnRNA/RNPs (Figure 3, lanes 3 through 6), while other sera, including the normal serum, reacted weakly with U1snRNP (Figure 3, lanes 7 and 8). The U1snRNA reactivity seems to better explain the cross-reactions obtained in ELISA using UsnRNPs as antigen and chagasic sera with higher absorbance values (Table 2) since these chagasic sera may have high affinity antibodies that can immunoprecipitate UsnRNPs. Anti-m3G antibody was used as a positive control to characterize the UsnRNPs in HeLa nuclear extracts (Figure 3, lane 9).

\section{Discussion}

Many of the specific host-parasite interactions in Chagas' disease may explain the tissue lesions of the peripheral nervous system observed in acute or chronic disease (6). Many T. cruzi antigens are involved in chronic chagasic cardiopathy, such as FL160-1, a surface protein associated with the $160 \mathrm{kDa}$ flagellum which has an epitope that cross-reacts with the nervous tissue, and Gp85, a surface glycoprotein which reacts with chronic chagasic sera as well as with common glycolipid antigens present both in the mammalian nervous system and in $T$. cruzi trypomastigote forms $(7,13,21,22)$. Experiments using molecular cloning have revealed that chronic chagasic cardiopathy 
patients present a strong humoral response against C-terminal regions of four cloned $\mathrm{P}$ ribosomal proteins, TcP1, TcP2, TcPJL5 and TcPo, which may be important in Chagas' disease immunopathology (10). Moreover, chronic serum antibodies recognized an antigen (43-45 kDa) in normal mouse heart and skeletal muscle tissue, suggesting that this glycoprotein may be a target for autoantibodies in Chagas' disease (11).

Fatenejad et al. (23) showed that the autoimmune response to snRNPs and possibly to other autoantigens in lupus is a specific reaction similar to that seen in a typical immune response to foreign antigens. The presence of autoantibodies has always indicated a complicated immune reaction, which cannot simply be explained by a direct participation of the target antigen. Several $T$. cruzi antigens present cross-reactivity with human tissues. Nevertheless, almost nothing has been reported thus far with respect to UsnRNPs. Studies carried out by Miatello and Fiorotto (24) demonstrated the existence of cross-reactivity between antibodies directed against the ribosomal antigen of the parasite and normal myocardial tissue of mice and rabbits after experimental infection or immunization with $T$. cruzi, thus suggesting the presence of infection-induced autoantibodies. Skeiky et al. (12) suggested that $T$. cruzi $\mathrm{P}$ proteins must contribute to the development of autoantibodies in chagasic patients and the appearance of anti-P autoantibodies may be explained by a similar mechanism in Chagas' disease and SLE. Solana et al. (25) demonstrated that chagasic patient sera reacted with human factors and parasite ribosomal antigens by ELISA and Western blot, although they did not present cross-reactivity with sera from patients with other infections or autoimmune diseases.

The present study confirmed the presence of autoantibodies in chagasic patients against heterologous UsnRNPs, which could be a consequence of epitope spreading from the initial parasite epitope. Experiments us- ing ELISA have indicated that it is easier to detect the presence of these autoantibodies by this methodology; however, they are difficult to visualize by immunoprecipitation assays. Interestingly, non-chagasic cardiac sera showed high and medium reactivity with $T$. cruzi antigen (lower absorbance) and also reacted with HeLa UsnRNPs (Tables 1 and 2). This suggests that individuals with heart disease of unknown etiology may develop autoimmune antibodies at any time, which could cross-react with $T$. cruzi. The Western blotting results showed that these non-chagasic cardiac sera reacted mainly with higher molecular weight $T$. cruzi epitopes (Figure 1, panel II), but presented faint bands with HeLa UsnRNPs as well as normal sera (Figure 2, lanes 3 and 4). The explanation for these findings is still unknown.

Since two primary hypotheses are proposed to account for pathogenesis in chronic T. cruzi infections (the persistence of the parasites at specific sites in the infected hosts causes chronic inflammatory reactivity and T. cruzi infection induces immune responses which are targeted at self tissues), our results support this autoimmune etiology, defining another putative autoantigen like snRNPs, but also argue in favor of the idea that Chagas' disease must be treated as a parasitic disease with efforts to enhance effective immune responses and reduce the parasite load. This is supported by the results with non-cardiac chagasic sera.

Chiale et al. (26) demonstrated a strong correlation between circulating anti- $\beta$-adrenergic receptor antibodies and ventricular arrhythmias in the setting of a structurally normal heart and in the presence of idiopathic cardiomyopathy and Chagas' heart disease; moreover, they observed that anti$\beta$-adrenergic receptor antibodies were rare in patients without ventricular arrhythmia. They also observed that antibodies directed against a ribosomal $\mathrm{P}$ protein of $T$. cruzi cross-reacted with and were stimulated by 
the B1-adrenergic receptors. This fact was attributed to a certain homology existing between the R13 peptide of the parasite and an epitope of the second extracellular loop of the $\beta 1$-adrenergic receptor.

Bach-Elias et al. (16) analyzed in chagasic sera the presence of autoantibodies directed against some fairly known autoantigens, Sm and RNP (here named antiUsnRNPs), which are typical of some autoimmune rheumatic diseases such as SLE and mixed connective tissue disease. The main reason to use these antigens was the fact that an external agent such as a retrovirus may initially be responsible for the development of anti-UsnRNP antibodies, mainly through the activation of antiviral antibody synthesis. These antibodies cross-react with a mimicry region in host UsnRNPs and may therefore trigger the onset of an autoimmune process. Three similar characteristics between Chagas' disease and some autoim- mune diseases such SLE may be mentioned: a) similar etiology, which means that an external agent may initiate an autoimmune process, b) presence of antibodies which recognize host proteins as foreign, and c) a chronic and generalized autoimmune process.

Then, the present results give more support to the previous explanations, since the autoantibodies in chronic chagasic patients were demonstrated here by using different methodologies. Similar studies using inbred mouse strains chronically infected with $T$. cruzi have been done by our group (Ambrósio DL, Castro LIR and Cicarelli RMB).

\section{Acknowledgments}

We thank Dr. Montserrat Bach-Elias (CSIC, Barcelona, Spain) for providing the UsnRNPs from HeLa nuclear extracts.

\section{References}

1. Szarfman A, Terranova VP, Rennard SI, Foidart JM, de Fátima Lima M, Scheinman JI \& Martin GR (1982). Antibodies to laminin in Chagas' disease. Journal of Experimental Medicine, 155: 11611171.

2. Acosta AM, Sadigursky M \& Santos-Buch CA (1983). Anti-striated muscle antibody activity produced by Trypanosoma cruzi. Proceedings of the Society for Experimental Biology and Medicine, 172: 364-369.

3. Schmunis GA (1987). Autoimmunity in Chagas' disease. Memórias do Instituto Oswaldo Cruz, 82 (Suppl 1): 287-310.

4. Levin MJ, Mesri E, Benarous R, Levitus G, Schijman A, Levy-Yeuati P, Chiale PA, Ruiz AM, Kahn A \& Rosenbaum MB (1989). Identification of a major Trypanosoma cruzi antigenic determinant in chronic Chagas' disease. American Journal of Tropical Medicine and Hygiene, 41: 530-538.

5. Sepulveda $P$, Liegeard $P$, Wallukat $G$, Levin MJ \& Hontebeyrie $M$ (2000). Modulation of cardiocyte functional activity by antibodies against Trypanosoma cruzi ribosomal P2 protein C terminus. Infection and Immunity, 68: 5114-5119.

6. Bonfa E \& Elkon KB (1986). Clinical and serological associations of the anti-ribosomal P protein antibody. Arthritis and Rheumatism, 29: 981-985.

7. Avila JL (1992). Molecular mimicry between Trypanosoma cruzi and host nervous tissues. Acta Cientifica Venezolana, 43: 330-340.

8. Eisen H \& Kahn S (1991). Mimicry in Trypanosoma cruzi: fantasy and reality. Current Opinion in Immunology, 3: 507-510.
9. Grauert MR, Houndayer M \& Hontebeyrie-Josckowiciz M (1993). Trypanosoma cruzi infection enhances polyreactive antibody response in an acute case of human Chagas' disease. Clinical and Experimental Immunology, 93: 85-92.

10. Levin MJ (1993). Humoral autoimmune response in Chagas' disease: Trypanosoma cruzi ribosomal antigens as immunizing agents. FEMS Immunology and Medical Microbiology, 7: 205-210.

11. McCormick TS \& Rowland EC (1993). Trypanosoma cruzi: recognition of a $43 \mathrm{kDa}$ muscle glycoprotein by antibodies present during murine infection. Experimental Parasitology, 77: 273-281.

12. Skeiky YA, Benson DR, Parsons M, Elkon KB \& Reed SG (1992). Cloning and expression of Trypanosoma cruzi ribosomal protein PO and epitope analysis of anti-PO autoantibodies in Chagas' disease patients. Journal of Experimental Medicine, 176: 201-211.

13. van Voorhis WC, Barrett L, Koelling R \& Farr AG (1993). FL-160 proteins of Trypanosoma cruzi are expressed from a multigene family and contain two distinct epitopes that mimic nervous tissues. Journal of Experimental Medicine, 178: 681-694.

14. Teixeira AR, Teixeira ML \& Santos-Buch CA (1975). The immunology of experimental Chagas' disease. IV. Production of lesions in rabbits similar to those of chronic Chagas' disease in man. American Journal of Pathology, 80: 163-180.

15. Cunha-Neto E, Duranti M, Gruber A, Zingales B, de Messias I, Stolf N, Bellotti G, Patarroyo ME, Pilleggi F \& Kalil J (1995). Autoimmunity in Chagas disease cardiopathy: biological relevance of a cardiac myosin-specific epitope crossreactive to an immunodominant Try- 
panosoma cruzi antigen. Proceedings of the National Academy of Sciences, USA, 92: 3541-3545.

16. Bach-Elias M, Bahia D, Teixeira DC \& Cicarelli RMB (1998). Presence of autoantibodies against small nuclear ribonucleoprotein epitopes in Chagas' patients' sera. Parasitology Research, 84: 796799.

17. Dignam JD, Lebovitz RM \& Roeder RG (1983). Accurate transcription initiation by RNA polymerase II in a soluble extract from isolated mammalian nuclei. Nucleic Acids Research, 1: 1475-1489.

18. Towbin H, Stachelin T \& Gordon J (1992). Electrophoretic transfer of proteins from polyacrylamide gels to nitrocellulose sheets: procedure and some applications. Biotechnology, 24: 145-149.

19. Steitz JA (1989). Immunoprecipitation of ribonucleoproteins using autoantibodies. Methods in Enzymology, 180: 468-481.

20. Cicarelli RMB, Castro LIR, Martins EC, Cegatti MB, Bahia D \& BachElias M (1998). Spliceosomal factors are recognized by chagasic sera. International Proceedings Division, Monduzzi Editore - 10th International Congress of Immunology, November 1-6, 1998. New Delhi, India, 1141-1145.

21. Travassos LR \& Almeida IC (1993). Carbohydrate immunity in Ameri- can trypanosomiasis. Springer Seminars in Immunopathology, 15: 183-204.

22. Gea S, Ordonez P, Cerban F, losa D, Chizzolini C \& Vottero-Cima E (1993). Chagas' disease cardioneuropathy: association of anti-Trypanosoma cruzi and anti-sciatic nerve antibodies. American Journal of Tropical Medicine and Hygiene, 49: 581-588.

23. Fatenejad S, Bennett M, Moslehi J \& Craft J (1998). Influence of antigen organization on the development of lupus autoantibodies. Arthritis and Rheumatism, 41: 603-612.

24. Miatello CS \& Fiorotto ER (1989). Ribosomal antibody response in rabbits and mice infected with Trypanosoma cruzi. Revista Argentina de Microbiologia, 21: 141-145.

25. Solana ME, Katsin AM, Umenzawa ES \& Miatello CS (1995). High specificity of Trypanosoma cruzi epimastigotes ribonucleoprotein as antigen in serodiagnosis of Chagas' disease. Journal of Clinical Microbiology, 33: 1456-1460.

26. Chiale PA, Ferrari I, Mahler E, Vallazza MA, Elizari MV, Rosenbaum MB \& Levin MJ (2001). Differential profile and biochemical effects of antiautonomic membrane receptor antibodies in ventricular arrhythmias and sinus node dysfunction. Circulation, 103: 1765-1771. 